\title{
Nanoencapsulation of Chromolaena odorata Extract Using Pluronic F127 as an Effectively Herbal Delivery System for Wound Healing
}

\author{
Ngoc-Dung Huynh Luu $\mathbb{D}^{1,2,3}$ Le Hang Dang $\mathbb{D}^{4,5}$ Hoang Minh Bui, ${ }^{6}$ \\ Trang Thuy Thi Nguyen, ${ }^{6}$ Bich Tram Nguyen, ${ }^{7}$ Le Son Hoang, ${ }^{2,3}$ and Ngoc Quyen Tran $\oplus^{4,5}$ \\ ${ }^{1}$ School of Medicine, Ho Chi Minh City, Vietnam \\ ${ }^{2}$ School of Biotechnology, International University, Ho Chi Minh City, Vietnam \\ ${ }^{3}$ Vietnam National University, Ho Chi Minh City, Vietnam \\ ${ }_{4}^{4}$ Institute of Applied Materials Science, Vietnam Academy of Science and Technology (VAST), Ho Chi Minh City, Vietnam \\ ${ }^{5}$ Graduate University of Science and Technology, Vietnam Academy of Science and Technology, HCMC, Vietnam \\ ${ }^{6}$ Faculty of Pharmacy, Nguyen Tat Thanh University, Ho Chi Minh City, Vietnam \\ ${ }^{7}$ Department of Natural Science, Thu Dau Mot University, Thu Dau Mot City 590000, Vietnam \\ Correspondence should be addressed to Ngoc Quyen Tran; tnquyen979@gmail.com
}

Received 15 December 2020; Revised 23 January 2021; Accepted 8 February 2021; Published 23 February 2021

Academic Editor: Linh Nguyen

Copyright $\odot 2021$ Ngoc-Dung Huynh Luu et al. This is an open access article distributed under the Creative Commons Attribution License, which permits unrestricted use, distribution, and reproduction in any medium, provided the original work is properly cited.

\begin{abstract}
Chromolaena odorata is a medicinal herb with prominent pharmacological properties. The therapeutic efficiency of Chromolaena odorata extracts and its ingredients have, however, been limited by various factors, including the lack of targeting capacity and poor bioavailability. To approach this drawback, ethyl acetate fraction extract of Chromolaena odorata- (EA.ChO-) encapsulated pluronic-based nanocarriers was disclosed herein. The most common pluronic triblock copolymer micelles (pluronic F127) was used for the nanosized formulation of Chromolaena odorata extract. The obtained results show that EA.ChO-encapsulated nanoparticles have a spherical morphology with a designed hydrodynamic size was about $183.7 \mathrm{~nm}$ and zeta potential $-39.5 \mathrm{mV}$. The EA.ChO nanoparticles are stable in different aqueous solutions (water, PBS 2.8, and PBS 7.4). The lyophilized form of the EA.ChO nanoparticles exhibited excellent stability for long-term storage. Notably, the EA.ChO nanoparticles were 1.3-1.4 fold more effective in the growth of fibroblast than the free EA.ChO, verifying the potential of pluronic F127 nanoparticles to the increased function of EA.ChO in the proliferation of fibroblast cell. In addition, bleeding stopped within $55 \pm 6 \mathrm{~s}$ which was $20 \mathrm{~s}$ faster than that of free EA.ChO and 38-44s faster than that of negative control treatments. The EA.ChO nanoencapsulation processed a rapid blood clot formation compared to control, free EA.ChO, pluronic F127, and water, suggesting the excellent bioavailability of EA.ChO nanoencapsulation. The obtained results thus provided a promising prospect for raising the activity Chromolaena odorata extract in wound healing application.
\end{abstract}

\section{Introduction}

Throughout the history, herbs have been used in healthcare improvement. Currently, herbal medicine has gained more attention due to their safety and promising pharmacotherapeutics in the Western medicine [1]. However, most of the natural compounds which are highly lipophilic are not ideal for drug delivery because they do not dissolve well in the body [2]. They have lower bioavailability and require repeated administration or higher doses in order to achieve the desired therapeutic effects, which can lead to acute toxicity, adverse effects, and low patient compliance $[2,3]$.

Nanoencapsulation technology is one of the most effective strategies to overcome the abovementioned hinders of herbal extract [3-6]. The use of nanoparticles as the herbal carrier has received a lot of attention with enthusiasm 
because it can help enhance the stability and the absorption of dug as well as the an effective permeability to the cell membrane resulting in the maximum of the therapeutic properties of herbal medicine [3]. As a matter of fact, various materials such as liposome, hydrogel has already been developed over the years for herbal medicine delivery purpose [2]. Amongst, polymeric nanoparticles are the most common selection for drug carriers because they are able to encapsulate drug inside nanocarrier due to their excellent biocompatibility, nontoxic to biological system, and biodegradation as well as high stability during storage $[4,7]$. In recent times, block copolymers have emerged as a potential agent for drug delivery and gene therapy [5]. The small size, unique nanoscopic architecture, stability, and ability of block copolymer micelles suitable for good compatibility with the drug of choice are all highly desirable characteristics for a drug delivery system [8]. One such block copolymer proposed for controlled drug delivery is pluronic (Poloxamer), which has a triblock PEO-PPO -PEO structure (PEO: poly(ethylene oxide); PPO: poly(propylene oxide)). At high temperatures, the central PPO block becomes hydrophobic, while the PEO blocks remain hydrophilic. Because of this amphiphilic nature, pluronic molecules, above a critical temperature and concentration, selfaggregate in aqueous solutions to form spherical micelles with hydrophobic PPO cores surrounded by hydrophilic PEO coronas $[5,9]$. Poloxamers generally regarded as nontoxic and nonirritant materials are used in a variety of oral, parenteral, and topical pharmaceutical formulations [10]. They are neither absorbed from the gastrointestinal tract nor metabolized in the body [11]. Poloxamer 407 (pluronic F127) is one of the most commonly used owing to its solubilizing capacity, low toxicity (LD50 in mice between $1.7 \mathrm{~g}$ and $5.0 \mathrm{~g} / \mathrm{kg}$ body weight), drug release characteristics, and compatibility with numerous biomolecules and excipients [12]. Poloxamer 407 is considered as an "inactive" ingredient for different types of preparations (e.g., IV, inhalation, oral solution, suspension, ophthalmic, or topical formulations) by FDA guide [11]. While there is an upsurge of reviews summarizing the applications of pluronic F127 in the delivery of synthetic drugs, the possible use of these materials in formulating herbal medicines has rarely been put to formal discussions in the literature.

Chromolaena odorata (L.) is a medicinal herb widely distributed in the tropical and subtropical areas. The leaves of the Chromolaena odorata $(\mathrm{ChO})$ are used in the traditional medicine for treatment of ailments such as cough, malaria fever, diarrhea, hemostasis, and wound healing [13]. Moreover, various pharmacological properties of ChO leaf extracts are reported such as antibacterial [14-17], anticancer [18, 19], anticonvulsant [20], antidiabetic [21-23], antidiarrheal $[24,25]$, antifungal [26, 27], anti-inflammatory [28-30], antioxidant [31-36], antiparasitic [37, 38], hemostatic and wound healing [39-42], and hepatoprotective activities [43, 44]. Pharmacological effects are attributed to the rich presence of lipophilic flavonoids of the leaves such as quercetin, sinensetin, sakuranetin, kaempferol, and salvigenin, which were isolated and identified [13]. However, the biggest dilemma allied with the use of these flavonoids is its low bioavailability due to poor aqueous solubility, which prevents them from clinical application. Therefore, improving solubility of the lipophilic flavonoids may improve the bioavailability and the overall pharmacological activity of $\mathrm{ChO}$ extracts.

In the present study, the ChO nanoencapsulation systems using pluronic F127 were first developed. This work describes our findings in relation to the potential usage of pluronic F127 as the carrier for the EA.ChO to promote the biological activity of this fraction. The morphology and size distribution of EA.ChO delivery system were characterized by dynamic light scattering (DLS) and transmission electron microscopy (TEM), respectively. The dynamic size of nanoencapsulation was investigated under different media as well as various storage times. Further, we reported some promising wound healing properties of the herbal delivery system, as evidenced by the proliferation of human fibroblast cells and the ability of hemostasis in vivo model.

\section{Materials and Methods}

2.1. Materials. Pluronic ${ }^{\circledR}$ F127 was supplied by Merk (Singapore). Folin and Ciocalteu's phenol reagents and gallic acid (standard reagent grade) were purchased at Sigma-Aldrich (Singapore). The other chemical agents for extraction step were ordered from Labscan (Thailand) or Chemsol VINA (Vietnam).

In cell culture, Dulbecco's modified Eagle's medium (DMEM), fetal bovine serum (FBS), penicillin-streptomycin solution $(10,000 \mathrm{U} / \mathrm{mL})$, and sodium bicarbonate originated from Sigma-Aldrich (Singapore). Phosphate-buffered saline (PBS) ( $\mathrm{pH} 7.4,1 \mathrm{X})$ was purchased from Gibco. Unless otherwise specified, all other chemicals were acquired from RCI Labscan and Alfa Aesar.

2.1.1. Plant Material. ChO leaves were collected from District 2, Ho Chi Minh City, Vietnam, in August 2018. The plant samples were identified by Doctor Van Hong Thien, Department of Biotechnology, Institute of Biotechnology and Food Technology. The voucher specimen (No. ChO-01) was deposited at School of Medicine-Vietnam National University at Ho Chi Minh City. The leaves were quickly rinsed, dried under shade for 7 days, and then ground into powder by a mechanic grinder and stored in the jugs until use.

2.1.2. Animal Material. Male Swiss albino mice $(20 \pm 2 \mathrm{~g})$ were obtained from the Institute of Vaccines and Medical Biologicals, Nha Trang (Vietnam). The mice were kept for at least 2 days before testing. All mice were fed with standard food and water. Experiments on mice were complied with International Guiding Principles for Biomedical Research Involving Animals [45]. All of the procedures in this research were approved by Scientific Council of School of Medicine-Vietnam National University, Ho Chi Minh City (Protocol M03).

2.2. Preparation of Plant Extracts. Powder of the leaves was extracted by ethanol $96 \%$ by using the percolation method. Then, the supernatant was concentrated by evaporating the solvent to collect the first crude extract. Next, it was dissolved in methanol $20 \%$ and sequentially shaken with different solvents such as petroleum ether, chloroform, and ethyl acetate 
(EA). The EA solution was collected. Finally, the EA solution was concentrated by evaporation, and the residue was further dried under vacuum at $40^{\circ} \mathrm{C}$ to obtain the EA crude fraction extract. It was stored in air-tight containers and preserved in the refrigerator for subsequent use.

2.3. Preparation of Nanoparticles. EA extract (1 g) was dissolved in ethanol. F127 (1 g) was prepared in deionized water $(20 \mathrm{ml})$ and then let at $4^{\circ} \mathrm{C}$ to reach the homogenous state. Extract solution was then dropwised into F127 which was under sonication. The obtained solution was further sonicated for 5 minutes. Next, the solution was stirred at room temperature overnight. The centrifugation at $21000 \mathrm{rpm}$ was applied to remove the nonencapsulated extract. The supernatant was collected and then freeze-dried for further study. The encapsulated efficacy was calculated via the ratio of total phenolic content in nanoparticles.

\subsection{Nanoparticle Characterization}

2.4.1. Morphology. The shape and surface morphology of the nanoparticles were analyzed by transmission electron microscopy (TEM) (JEM-1400 JEOL).

\subsubsection{Analysis of Particle Size and Particle Size Distribution.} The $Z$-average particle size and particle size distribution of nanoparticles were analyzed using nanopartica SZ-100 series instrument (Horiba). For size measurement, the lyophilized form of the EA.ChO nanoparticles was redissolved into PBS 7.4 (1X, Gibco) to make $100 \mathrm{ppm}$. The solution was kept at $4^{\circ} \mathrm{C}$ for $24 \mathrm{~h}$ before examination. The hydrodynamic size of nanoparticles was recorded under following condition: $\theta=$ $90^{\circ}$, temperature $=25^{\circ} \mathrm{C}$, and gate time $=0$ to $1024 \mathrm{~ms}$. The size of nonencapsulated pluronic F127 was prepared with similar protocol of EA.ChO nanoparticles.

2.4.3. Measurement of Zeta Potential. The zeta potential of the nanoparticle was measured using nanopartica SZ-100 series instrument (Horiba). EA.ChO nanoparticles were prepared in PBS 1X (pH 7.4, Gibco) and then were let stable at $4^{\circ} \mathrm{C}$ before investigation. This obtain solution was loaded into electrophoretic cell. The data was obtained at $25^{\circ} \mathrm{C}$.

2.5. Stability Test of Nanoparticles. First, EA.ChO was dissolved in various aqueous conditions (water, PBS 2.8 and PBS 7.4). Then, these solutions were stable at room temperature for $24 \mathrm{~h}$ before measuring size-based DLS techniques. Second, EA.ChO (100 ppm) in PBS 7.4 was put in the room temperature for different time points. At the determined time, the size of EA.ChO was detected under the same condition with previous DLS testing. Each experiment was repeated at 3 independent times.

2.6. In Vitro Release Study. The in vitro drug release was studied by dialysis bag diffusion method [46]. $1 \mathrm{ml}$ sample (EA extract or EA.ChO nanoparticles) was dispersed into dialysis bag $(3.5 \mathrm{kDa})$, and the dialysis bag was immersed in $20 \mathrm{ml}$ of PBS $1 \mathrm{X}$ at $37 \pm 0.5^{\circ} \mathrm{C}$ with continuous magnetic stirring at $60 \mathrm{rpm}$. Samples $(1 \mathrm{ml})$ were withdrawn at a defined time intervals and replaced with equal amounts of fresh PBS 1X to maintain a constant volume. After suitable dilutions, the samples were determined the phenolic contents in the free EA extract and EA.ChO nanoparticles and the cumulative percentage of phenolics released was calculated by the following formula: cumulative percentage of phenolics released $(\%$ )$=($ cumulative amount of phenolics in dissolution medium at the predetermined time)/total amount of phenolics in EA. ChO nanoencapsulation $) \times 100 \%$.

2.7. Determination of Phenolic Contents. The total phenolic content was determined for individual extracts using the Folin-Ciocalteu method [47]. Briefly, $0.5 \mathrm{~mL}$ of extract solution was mixed with $2.5 \mathrm{~mL}$ of $0.5 \mathrm{~N}$ Folin-Ciocalteu reagent and incubated at $37^{\circ} \mathrm{C}$. After 4 minutes, $2.2 \mathrm{~mL}$ of $\mathrm{Na}_{2} \mathrm{CO}_{3}$ (10\%) was subsequently added to the mixture and incubated at $37^{\circ} \mathrm{C}$ for 2 hours with intermittent agitation. Afterwards, the absorbance was measured utilizing a UV Spectrophotometer (Shimazu, UV-1800) at $760 \mathrm{~nm}$ against a blank without extract. The outcome data were expressed as $\mathrm{mg} / \mathrm{g}$ of gallic acid equivalents in milligrams per gram (mg GAE/g) of dry extract.

\subsection{In Vitro Cytotoxicity and Cell Proliferation Study}

2.8.1. Cytotoxicity Assay. The SRB assay [48] was used to carry cytotoxicity in cell-based studies. Human fibroblast cells (BJ (ATCC ${ }^{\circledR}$ CRL-2522 ${ }^{\mathrm{TM}}$ )) were seeded on 96-well plates with the density of $2 \times 103$ cell/well and cultured in DMEM supplemented with $10 \%$ FBS and $1 \%$ penicillin and streptomycin at $37^{\circ} \mathrm{C}, 5 \% \mathrm{CO}_{2}$, and $90 \%$ humidity for $24 \mathrm{~h}$ before incubated with testing samples. The EA.ChO nanoparticles were prepared in water while the raw EA extract was prepared in DMSO, and then diluted with fully supplement DMEM to reach different concentration of the samples (from 0.025 to $1 \mathrm{mg} / \mathrm{ml}$ ). Water and DMSO were used as the controls in this study. At the determined time points $(48 \mathrm{~h}$, $72 \mathrm{~h}$, and $96 \mathrm{~h}$ ), Sulforhodamin B Assay Kit (ab235935) was used to determine the cell viability following the guidance of manufacturer.

2.8.2. Cell Proliferation. First, $2 \times 104 \mathrm{cell} / \mathrm{ml}$ was placed in the $35 \mathrm{~mm}$ culture dishes, and the completed DMEM (supplemented with $10 \%$ FBS and $1 \%$ penicillin and streptomycin) was added. After $24 \mathrm{~h}$ incubated at $5 \% \mathrm{CO}_{2}$ and $90 \%$ humidity, the media was discarded and the new media containing various concentrations of samples was supplied. These cells were then put in incubator at the same condition. At the determine time, the media was removed and the cell was washed 3 times with PBS $1 \mathrm{X}$ before adding $0.25 \%$ Trypsin-EDTA (Gibco). The cells were then collected via the centrifugation at $1200 \mathrm{rpm}$. Full DMEM was added in the pellet cell. $10 \mu \mathrm{l}$ of this suspension was mixed with $10 \mu \mathrm{l}$ trypan blue $(0.4 \%)$ and then loaded into hemacytometer. The results were reared by cell counter machine (Countess ${ }^{\circledR}$ Automated Cell Counter-life technologies). The growth rate was calculated via the below formula:

$$
\text { Growth rate }=\left(\frac{\text { treated cell }}{\text { nontreated cell }}\right) \times 100 \text {. }
$$


The experiment was repeated 3 times for each independent replication.

2.9. In Vivo Hemostatic Activity Test. The mice were divided into 4 groups: group I (EA.ChO nanoparticle) and group II (EA extract) were treated groups while group III (F127) and group IV (water) were kept as control groups. After 21 consecutive days, the bleeding time and clotting time test were done.

2.9.1. Bleeding Time. Bleeding time was based on the method described by Rajasekaran [49]. Bleeding time was assessed by cutting the tip of the tail of each mouse with a sharp pair of scissors $(5 \mathrm{~mm})$; then, the tail was placed in an isotonic saline solution maintained at $37^{\circ} \mathrm{C}$ immediately. A stopwatch was started simultaneously with the immersion of the tail in the saline solution. Bleeding time was noted from appearance of the first drop of blood to the bleeding stopped completely.

2.9.2. Clotting Time (Drop Method) [50]. First, place a drop of blood ( $5 \mathrm{~mm}$ in diameter) from the tail on a dry glass slide. Then, start the stop watch and note the time. Next, draw a pin through the drop every 30 seconds, and note the time when fibrin threads adhere to the pin and move with it out to the blood drop. The time interval between placing the blood drop on the slide and the formation of fibrin threads was taken as the clotting time.

2.10. Statistics. Results were expressed as mean \pm SEM (standard error of the mean). Statistical difference between groups was determined using Welch's $t$-test. A $p$ value of $<0.05$ was considered statistically significant.

\section{Results and Discussion}

3.1. Characterization of Chromolaena odorata Nanoencapsulation Systems. In the present study, ethyl acetate fraction extract of the leaves of Chromolaena odorata (EA.ChO) was encapsulated into pluronic F127 micelles by a nanoprecipitation technique combining ultrasonic emulsification and solvent evaporation. The size distribution for empty F127 micelles and EA.ChO loaded micelles are illustrated in Figure 1. The hydrodynamic size of empty F127 micelles when dissolved in PBS at $\mathrm{pH} 7.4$ during preparation was $10.6 \mathrm{~nm}$ with polydispersity index (PDI) 0.499 (Figure 1(a)). The incorporation of EA.ChO increases the radii of the micelles, which is parallel with previous experiments involving the encapsulation of drug molecules in pluronic block copolymer micelles $[51,52]$. After incorporating EA.ChO, the average size of nanoparticles was $183.7 \mathrm{~nm}$ (Figure 1(b)), suggesting the evidence for the successful loading process. In addition, the time correlation function of EA.ChO nanoparticles (inset in Figure 1(b)) exposed a single exponential decay riding on top of a baseline, indicating that EA.ChO nanoparticles are homogeneous size distribution [53] and the interaction between these nanoparticle-like aggregates are small. Nanoparticles of such sizes are known to be easily uptaken by cells [54] rather than eliminated in the blood stream [55], which constitutes a promising feature of EA.ChO nanoparticles in the light of their biomedical application. In agreement with this, the zeta potential was determined to $-39.5 \mathrm{mV}$ (Figure $1(\mathrm{c})$ ), which was indicated the high stability of nanoparticles [56]. The negative value in zeta potential could be due to negative charge of phenolic compounds at the surface of particles (F127 micelles) [57] or a higher exposure of negative functional groups of EA.ChO when interacting with incorporated phenolics [58]. Further, the TEM images (Figure 1(d)) showed spherical particles with uniform size and with homogenous structure, which is consistent with the previous assumption about their time correlation function. Regarding drug delivery systems (DDSs), drug-loading efficacy is a critical parameter that directly affects the therapeutic efficacy of the system. Herein, taking total phenolic content (calculated by Folin-Ciocalteu assay) as the evaluation index, the encapsulation efficiency (EE) was established as $81.4 \pm 1.26 \%$, corresponding to $142.5 \pm 1.24$ GAE micrograms per milligram of EA.ChO nanoparticles $(1 \mathrm{mg})$.

3.2. Stability of EA.ChO Nanoencapsulation Systems. The stability of polymeric nanostructured systems plays a critical factor to identify the potential application of this system in biomedical fields. First, the hydrodynamic size (Figure 2) of EA.ChO nanoparticles was assessed to provide their physicochemical behavior in some aqueous solutions (water and PBS $1 \mathrm{X}$ with $2 \mathrm{pH}$ values). The evolution of EA.ChO nanoparticle size in water or PBS was almost similar and did not register significant changes. The hydrodynamic size of EA.ChO was around $180 \mathrm{~nm}$ and small PDI value (PDI value $<0.25$ ), showing that EA.ChO nanoparticles were of the good dispersed quality in these aqueous solutions.

For additional study in stability, EA.ChO nanoparticles were incubated in PBS $1 \mathrm{X}$ and $\mathrm{pH} 7.4$ to simulate physiological conditions, and DLS was monitored over time. The DLS data for EA.ChO nanoparticles at different storage time points are presented in Figure 3. In agreement with zeta potential value of EA.ChO nanoparticles, no statistically significant differences were observed in the size distribution of the aggregates as determined by DLS over a period of 35 days (Welch's $t$-test, $p>0.05$ ). The size of EA.ChO nanoparticles was $179.4 \mathrm{~nm}, 178.6 \mathrm{~nm}, 181.4 \mathrm{~nm}$, and $182.2 \mathrm{~nm}$ with a narrow distribution at $2,12,22$, and 35 days, respectively. Moreover, the good stability of EA.ChO nanoparticles was also exposed through the behavior of their time correlation function (inset of Figures 3(a)-3(d)). All the autocorrelation function profiles at each determined time points were in sigmoidal behavior. In addition, EA.ChO nanoparticles' correlograms appeared to decay around the same time point, indicating comparably sized particle populations for all time points. Moreover, they also exhibited similar gradients, suggesting comparably polydisperse particle populations.

3.3. In Vitro Drug Release. The release profiles of total phenolic contents from EA.ChO extract and EA.ChO nanoparticles were evaluated in vitro as a function of time in physiological buffer PBS (1X, pH 7.4) at $37^{\circ} \mathrm{C}$ (Figure 4). The release profiles of both raw EA.ChO extract and its nanoparticle-based pluronic F127 followed a similar behavior with an initial burst release in first 30 minutes; however, thereafter, there 


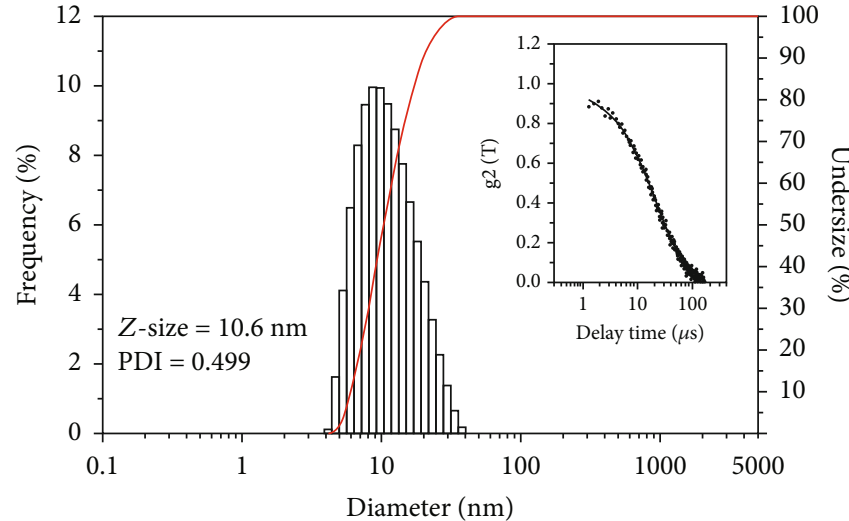

(a)

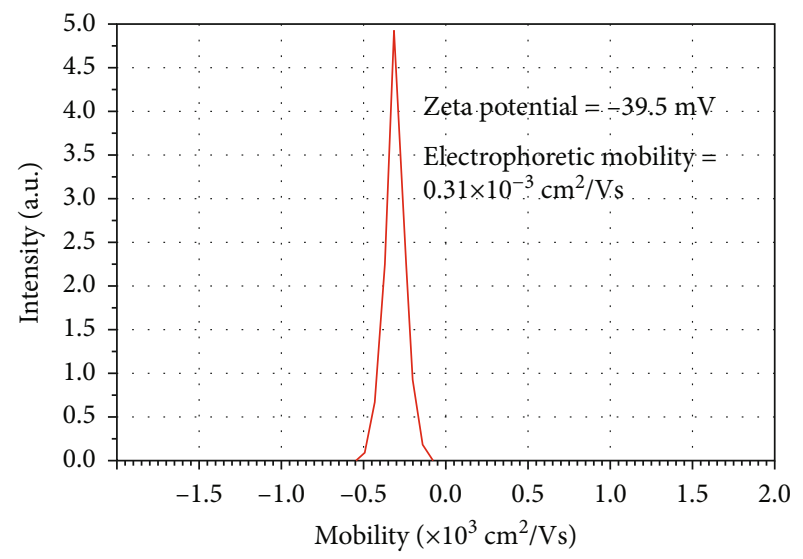

(c)

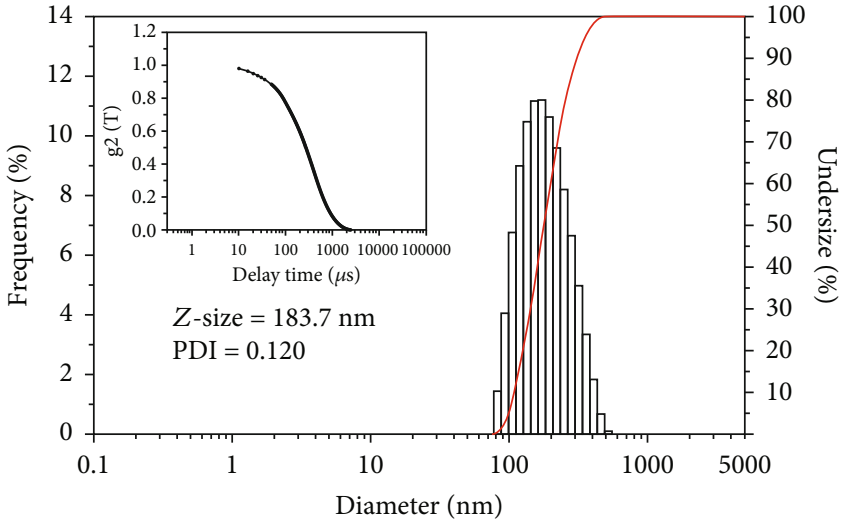

(b)

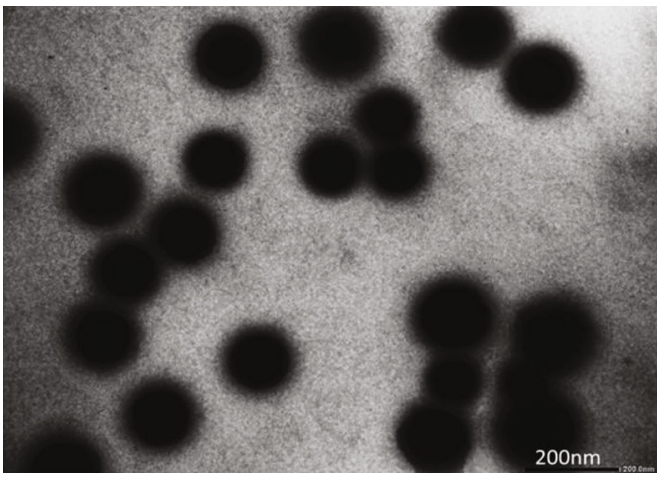

(d)

Figure 1: (a) Hydrodynamic size of pluronic F127 nanoparticles (100 ppm, PBS) at $25^{\circ} \mathrm{C}$. (b) The size, (c) potential change, and (d) TEM image of nanoparticles after encapsulating EA extract (100 ppm, PBS). Time correlation function is inserted into sized distribution.

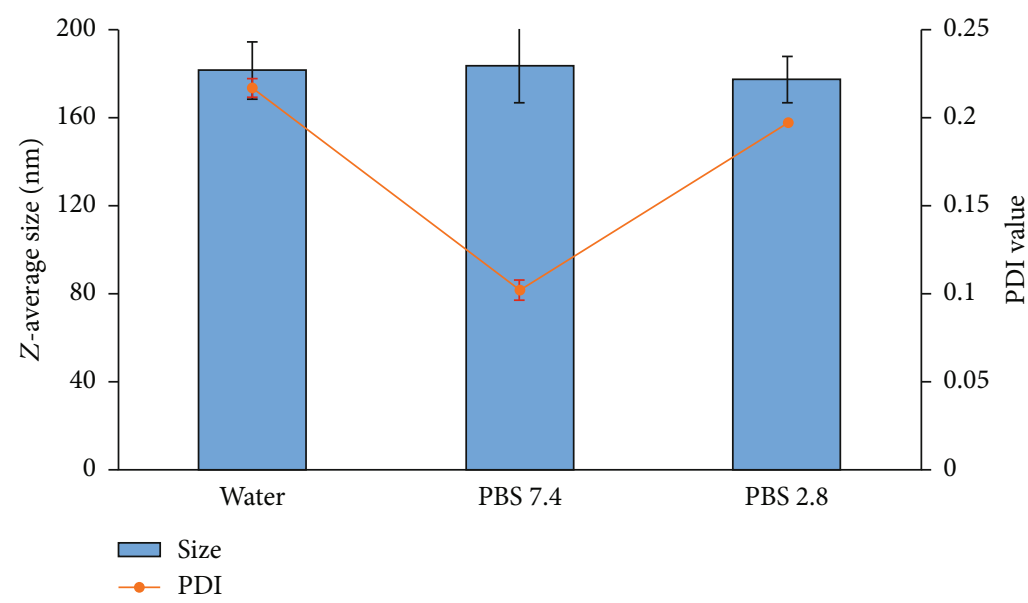

FIGURE 2: The hydrodynamic diameter of EA.ChO nanoparticles at different aqueous solutions: pure water, physiological buffer PBS $1 \mathrm{X}$ (pH 7.4 and $\mathrm{pH} 2.8$ ).

was remarkable discrepancy between the two variations. The release of phenolic compounds from as-prepared EA.ChO extract presented a more pronounced initial burst effect, reaching approximately $54.99 \pm 7.73 \%$ of phenolic compounds released in 30 minutes, whereas only $21.29 \pm 0.42 \%$ of phenolic compound was released from nanoparticles. The amount of phenolic compounds from raw extract samples accumulating in the medium was accelerated and then reached nearly $100 \%$ by the end of $12 \mathrm{~h}$. On the contrary, after initial burst release, the percentage of phenolic 


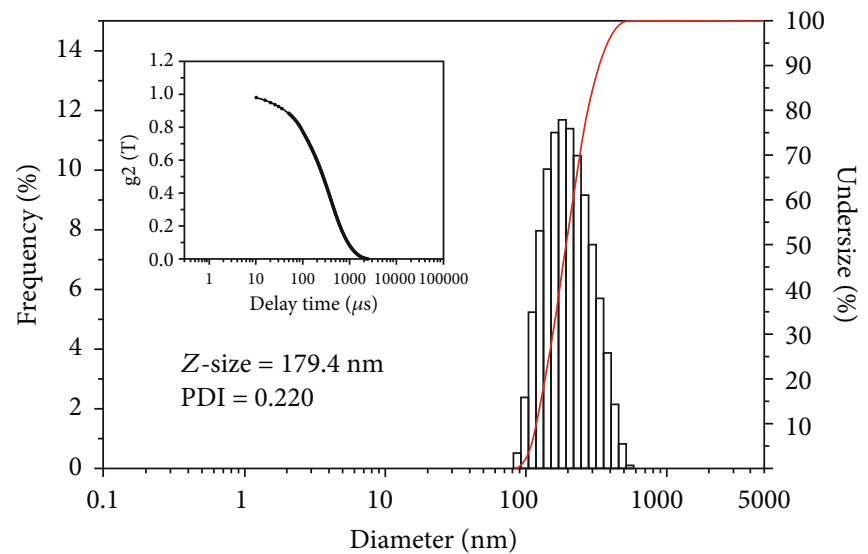

(a)

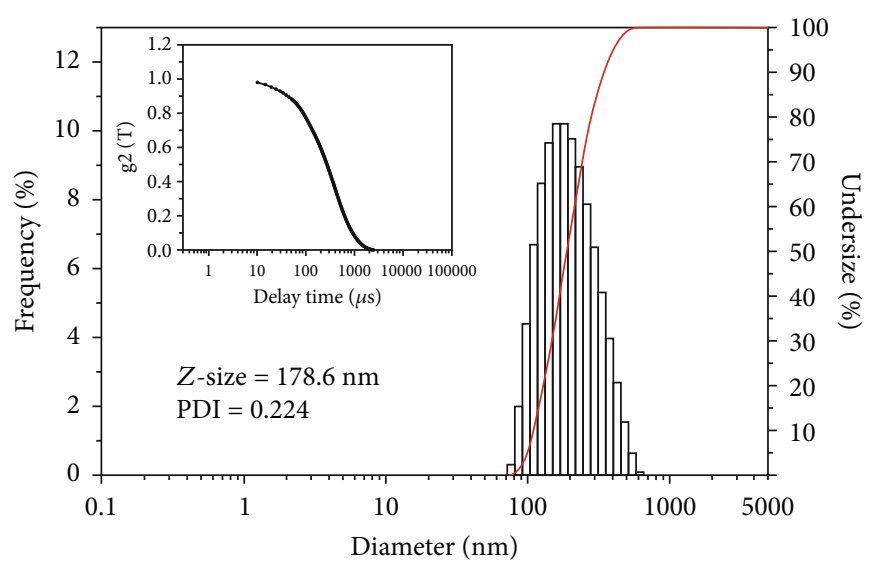

(b)

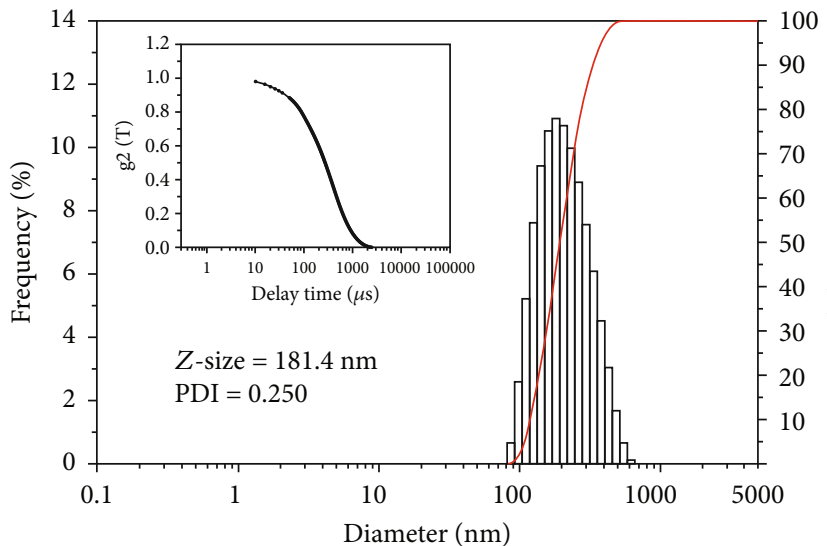

(c)

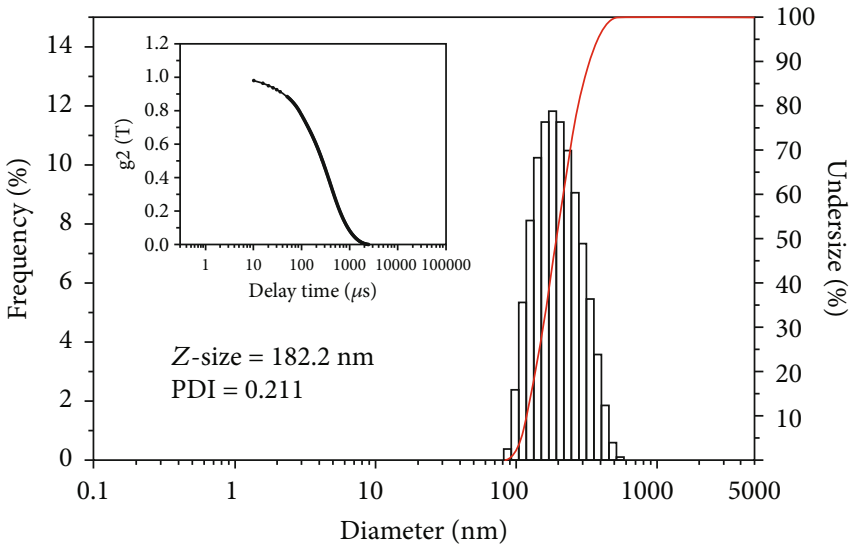

(d)

Figure 3: Time correlation function and the hydrodynamic diameter of EA.ChO nanoparticles after different storage times: (a) day 2, (b) day 12 , (c) day 22, and (d) day 35. Time correlation function is inserted into sized distribution.

compounds releasing in the medium was gradually increased to $71.07 \pm 2.93 \%$ over period of $96 \mathrm{~h}$, exhibiting the slow and sustained release of phenolic compound from EA.ChO extract at physiological $\mathrm{pH}$.

3.4. Effects of Chromolaena odorata Leaf Extract and Its Nanoencapsulation on the Viability of Fibroblast Cells. The concentration-dependent cytotoxicity of EA.ChO nanosys- tem based on F127 micelles was examined with human fibroblast after difference in time of exposure ( $48 \mathrm{~h}, 72 \mathrm{~h}$, and $96 \mathrm{~h}$ ) and compared to that of free EA.ChO extract. Blank-pluronic F127 nanoparticles had no effects on the fibroblast cell viability and showed a similar result to the nontreated cells. It can be inferred that the pluronic F127 nanoparticles are biocompatible and suitable for usage in biological applications. As shown in Figure 5, the cytotoxic effects of free EA.ChO were 


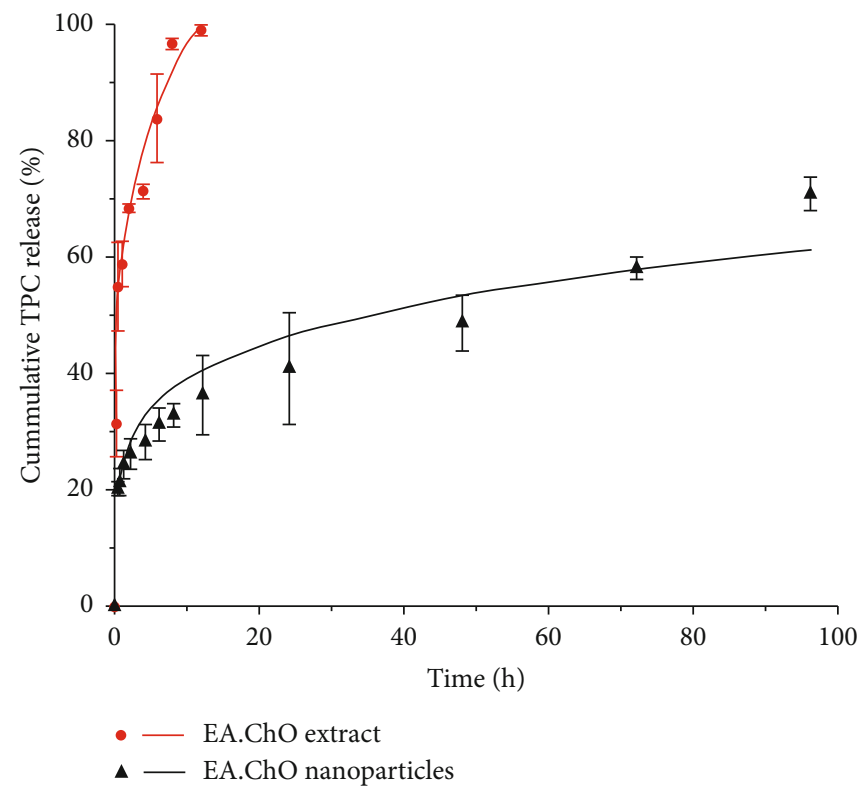

FIGURE 4: Cumulative release profiles of total phenolic contents from EA.ChO extract and EA.ChO nanoparticles as a function of time in PBS $1 \mathrm{X}, \mathrm{pH}$ 7.4. Error was SEM values (standard error of mean) calculating from 3 observations.

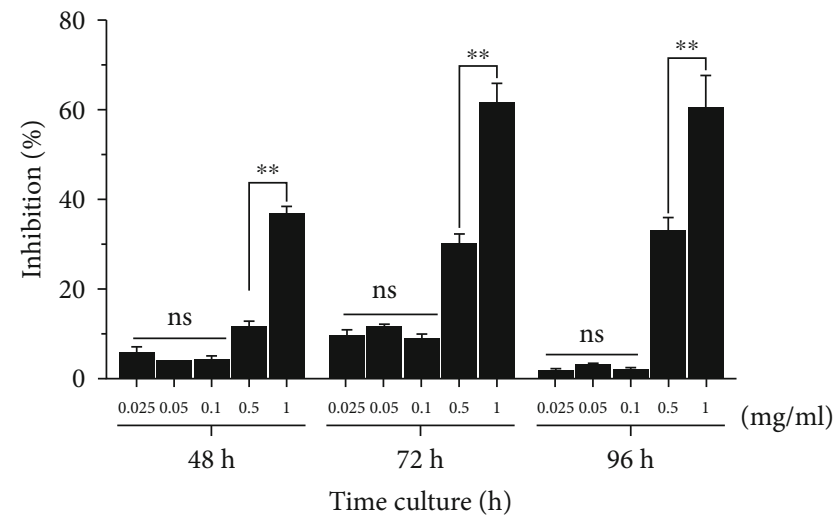

(a)

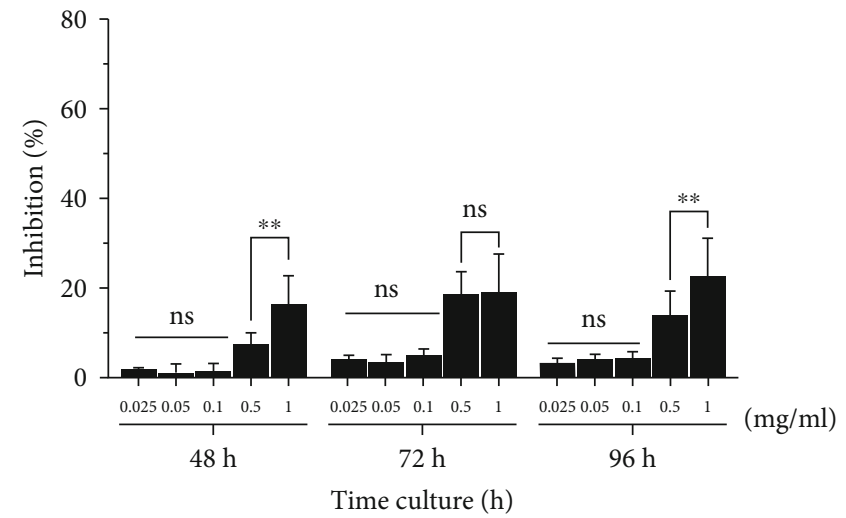

(b)

Figure 5: The effect of series dose of (a) EA.ChO extract and (b) EA.ChO nanoparticles on the viability of human fibroblast cells at different culture time: $48 \mathrm{~h}, 72 \mathrm{~h}$, and $96 \mathrm{~h}$. Data was presented as mean \pm SEM of three independent replications. ${ }^{* *} p<0.01$ while "ns" means nonsignificance.

remarkably higher than EA.ChO nanoparticles as the expectation. While the very low toxicity percent (below 6\%) obtained in the first $48 \mathrm{~h}$ incubation with concentration in range of 0.025 to $0.1 \mathrm{mg} / \mathrm{ml}$, at relatively high concentrations of free EA.ChO extract (up to $1 \mathrm{mg} / \mathrm{ml}$ ), the mortality was remarkably increased to $37.25 \pm 1.27 \%$. In the contrary, despite the dose-dependent cytotoxicity of EA.ChO nanoparticles, after $48 \mathrm{~h}$ incubation, the percentage of inhibition of fibroblast cell growth was lower than that of free EA.ChO extract even at the highest concentration $1 \mathrm{mg} / \mathrm{ml}$ $(16.49 \pm 6.41 \%$ cell dead). Further extension of the incubation time, the concentration of testing sample that induces the diminishing of the growth of cells by $50 \%\left(\mathrm{IC}_{50}\right)$ was identified for EA.ChO extract in the range of tested concentration, $0.73 \pm 0.07 \mathrm{mg} / \mathrm{ml}$ (at $72 \mathrm{~h}$ ) and $0.71 \pm 0.05 \mathrm{mg} / \mathrm{ml}$ (96h). Our results with free EA.ChO extract were totally agreed with the previous reports $[59,60]$. In a significant reversal of free fractions, $\mathrm{IC}_{50}$ was indeterminate for EA.ChO nanoparticles at $72 \mathrm{~h}$ and $96 \mathrm{~h}$, confirming the nontoxic properties of EA.ChO nanoparticles. The obtained results signified that EA.ChO extract encapsulated in pluronic F127 micelles produces in a lower safety risk for the EA.ChO therapy. This may be due to the control of release of phenolic compound of EA.ChO extract leading to reduce the risk of the quick increase of the cellular level of reactive oxygen species (ROS), consequently, in the lower cytotoxicity of the EA.ChO nanoencapsulation compared to the native EA.ChO.

\subsection{Effects of the EA.ChO Nanoencapsulation Systems on the} Proliferation of Fibroblast Cells. The proliferation of fibroblast cell is indispensable in wound repair, particularly in 


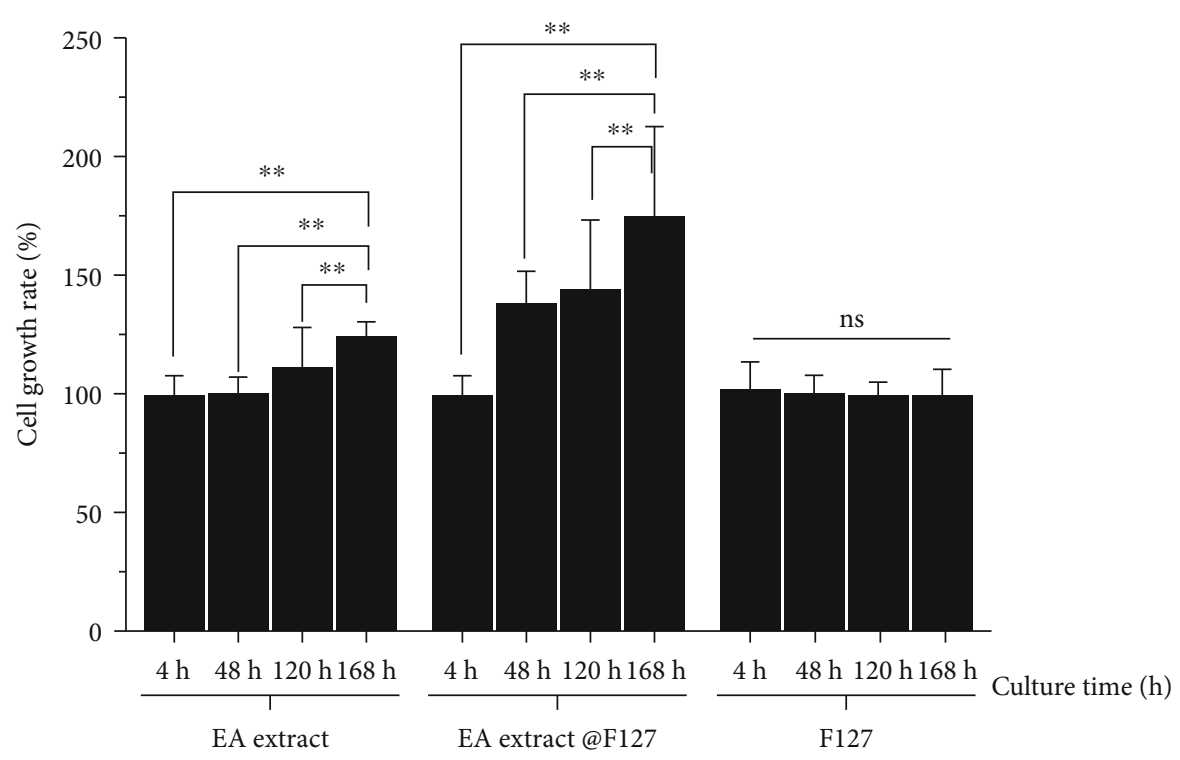

Figure 6: The growth rate of fibroblast cell culturing with different variables (EA.ChO extract, EA.ChO nanoencapsulation, and bare pluronic F127) at different time points $(4 \mathrm{~h}, 48 \mathrm{~h}, 120 \mathrm{~h}$, and $168 \mathrm{~h})$ as compared to nontreated cells. Results are presented as mean \pm SEM of 3 independent replications. ${ }^{* *} p<0.01$; ns: nonsignificant difference.
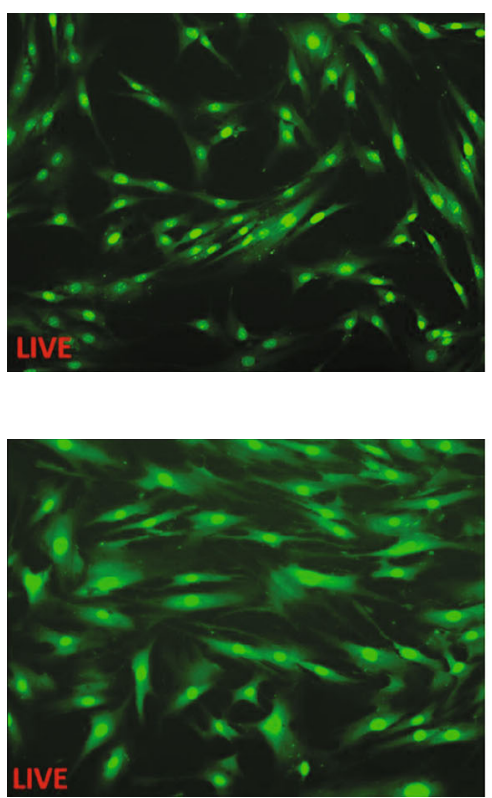

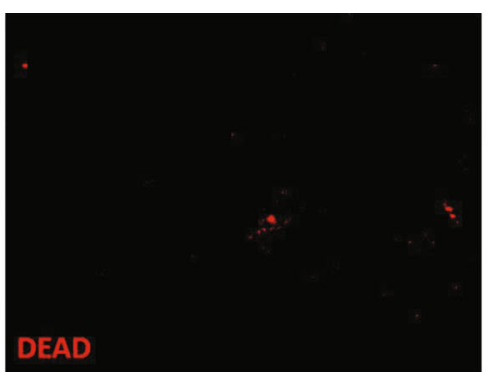

(a)

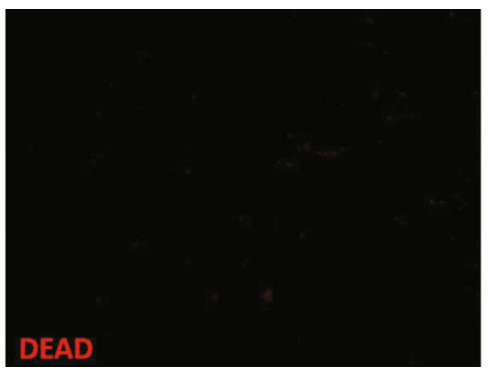

(b)
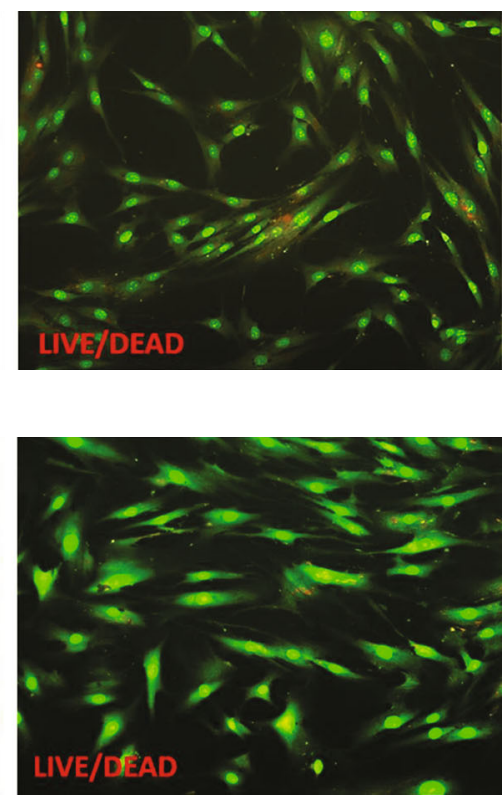

Figure 7: Fluorescent microscopy images of human fibroblast cell grown for $168 \mathrm{~h}$ in the presence of (a) control and (b) $0.1 \mathrm{mg} / \mathrm{ml}$ EA.ChO nanoparticles. Scale bar: $100 \mu \mathrm{m}, 100 \mathrm{x}$.

the early phase of healing process. Stimulation of their proliferation is one mechanism by which an agent might enhance the repair. To predict the potential application of EA.ChO nanoparticles in wound healing, human fibroblast cells were selected as the model for proliferation assay. Based on the cytotoxic results, the concentration of EA.ChO extract as well as EA.ChO nanoparticles selecting in this study were at $0.1 \mathrm{mg} / \mathrm{ml}$. As shown in Figure 6, the growth rate of fibroblast cells incubating with bare F127 nanoparticles $(1 \mathrm{mg} / \mathrm{ml})$ and controls (untreated cells) was inconsequential different over a period of $168 \mathrm{~h}$ (all $p$ value $>0.1$ ). For EA.ChO extract, the stimulation of fibroblast growth only reached significance after $120 \mathrm{~h}$ and was $111.59 \pm 16.68 \%$ of the growth of control $(p<0.01)$. By $168 \mathrm{~h}$ incubation with $0.1 \mathrm{mg} / \mathrm{ml} \mathrm{EA.ChO}$ extract, the growth of fibroblast cells enhanced up to 125.13 $\pm 6.04 \%$ of that of control cells $(p<0.01)$. A significant enhancement $(p<0.01)$ of the growth of fibroblast cell incubated with EA.ChO nanoparticles was evident as early as $48 \mathrm{~h}$, about $137.95 \pm 14.31 \%$ regarding untreated cells. In addition, the proliferation rate of fibroblast cells stimulating 


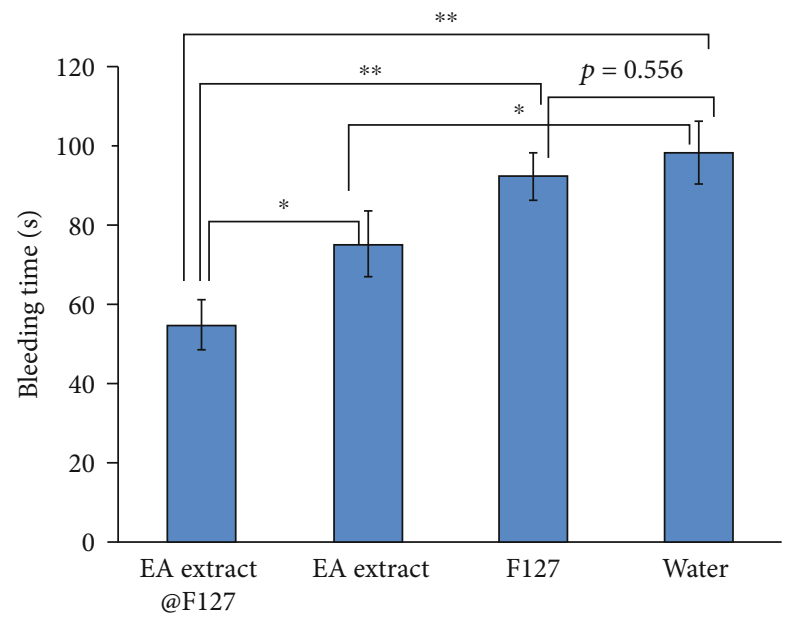

(a)

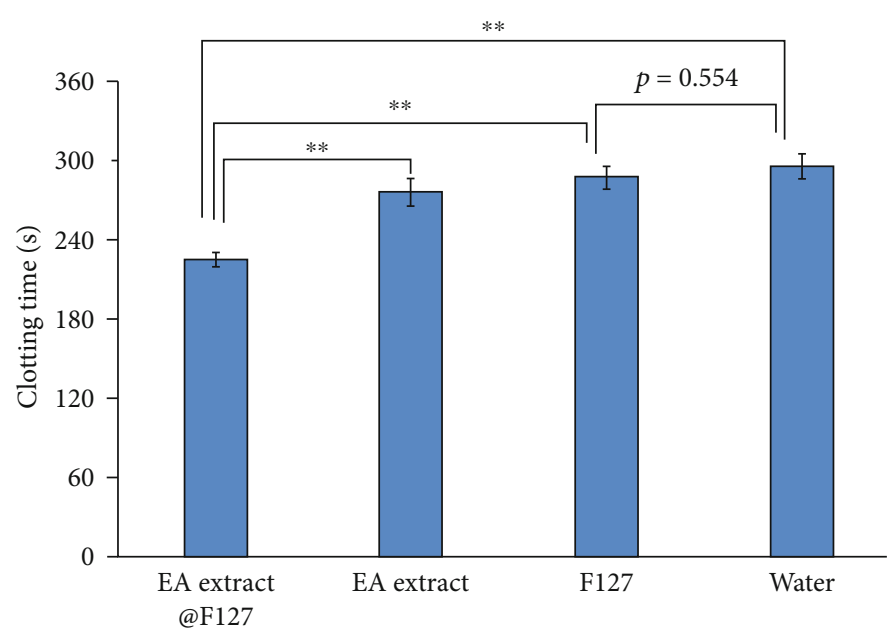

(b)

FIGURE 8: Hemostatic performance of EA.ChO extract and its nanoformulation form through (a) bleeding time and (b) clotting time on mice model as compared to control treatments (water and F127). Data were shown as mean \pm SEM; ${ }^{*} p<0.05,{ }^{* *} p<0.01$, and $n=6$.

by EA.ChO nanoparticles was strongly positive increase at all subsequent time points. After $120 \mathrm{~h}$ stimulating with EA.ChO nanoparticles, the growth rate of fibroblast was $144.22 \pm 29.23 \%$ of that in DMEM media and was 1.3 times as compared to EA.ChO extract $(p<0.01)$. The degree of stimulation relative to fibroblast cell in nontreated cells increased to $175.12 \pm 38.47 \%$ by $168 \mathrm{~h}$ culture. The density of fibroblast in case of EA.ChO nanoparticles increased 3.35 times of that on first $4 \mathrm{~h}$ culture $(p<0.01)$ and was 1.4 times higher than that in terms of free EA.ChO extract $(p<0.01)$.

Observation by fluorescent microscopy (Figure 7) showed that the morphology of fibroblast cells incubating with EA.ChO nanoparticles $(0.1 \mathrm{mg} / \mathrm{ml})$ displays typical spindle-like shape as similar to control cells. Although the number of seeding cells was identical in all tested variables, the density of cells was higher in EA.ChO nanoparticles than in the control. Moreover, cell size is also bigger than control. The fibroblast in EA.ChO nanoparticles was in large and well spread. This indicates that the fibroblast cell was metabolically active in the presence of the proposed nanoformulation of EA.ChO.

3.6. In Vivo Hemostatic Activity. In the wound healing process, cessation of bleeding is the first step [61, 62]. Various studies have been proved that $\mathrm{ChO}$ leaf extract promoted the excellent hemostatic activity [42, 63]. In this study, our objective was to investigate whether EA.ChO nanoparticles can improve the effectiveness to stop bleeding, compared to raw EA.ChO extract. Following the previous report [64], the dose $150 \mathrm{mg} / \mathrm{kg}$ body weight was selected. For the first screening of acute toxicity, both EA.ChO extract and its nanoformulation at this dose were nonlethal for mice, and the behavior and activity were normal in treated mice even after 21-day administration.

As demonstrated in Figure 8, there was no significant difference in both bleeding time $(p=0.556>0.05)$ and clotting time $(p=0.554>0.05)$ between pluronic F127 and watertreated mice. Under the identical condition, EA.ChO nanoparticle-based pluronic F127 significantly reduced bleeding time and clotting time compared to both control samples (all $p<0.01$ ) while EA extract just slightly reduced the bleeding time compared to the water group $(p<0.05)$. The hemorrhage in the mice treated with EA.ChO extract had stopped after $74.83 \pm 8.28 \mathrm{~s}$, whereas the coagulant activity of EA.ChO nanoparticles was remarkably improved, about $20.16 \pm 2 \mathrm{~s}$ and $50.8 \pm 4.93 \mathrm{~s}$ quicker than that of raw extract in stopping blood and coagulation time, respectively. These results further confirm that EA.ChO nanoparticles have excellent hemostatic activity in vivo. The improvement in controlling the bleeding and coagulation time of EA.ChO nanoparticles may due to the enhancement of the absorption of bioactive compounds in EA.ChO extract.

\section{Conclusion}

Considering the potential applications of Chromolaena odorata leaf extracts in wound healing, this work developed and demonstrated a strategy to improve their functionality, stability, and cytotoxicity as well as bioavailability of the EA.ChO extract via pluronic F127 micelle-based nanoencapsulation. The EA.ChO extract-loading pluronic F127 has spherical shape, and the dynamic size was below $200 \mathrm{~nm}$, proposing the good absorption in the in vivo application. The size of EA.ChO nanoparticles was also resistant with the change of $\mathrm{pH}$ of aqueous solutions. Furthermore, the EA.ChO nanoparticles were stable at prolonged duration of storage time. The comparison of the release profiles of both raw extract and its nanoformulation form revealed the more suitable strategy of utilizing pluronic F127 to encapsulate EA.ChO extract. In vitro cytotoxic assay using fibroblast cell found that the toxicity of EA.ChO extract was significantly reduced as encapsulated in pluronic F127 nanocarriers. Notably, the nanoencapsulation of EA.ChO extract greatly enhanced the proliferative activity of fibroblast cell. In addition, the EA.ChO extract nanoformulation showed an improved blood clotting ability and a reduced blood bleeding 
in vivo assays as compared to raw extract. In summary, results in this study showed that the polymeric nanoparticle-based pluronic F127 could be used as a strategy to enhance herbal extract bioactivities and present potential for further investigations in food systems and pharmaceutical applications.

\section{Data Availability}

All data used to support the findings of this study are included within the article.

\section{Conflicts of Interest}

The authors declared no conflicts of interest regarding the publication of this paper.

\section{Acknowledgments}

This research is funded by Viet Nam National University Ho Chi Minh City (VNU-HCM) under grant number (C201944-01). The authors would like to thank Doctor Van Hong Thien, Department of Biotechnology, Institute of Biotechnology and Food Technology, Ho Chi Minh City, Vietnam, for authenticating the plant samples. The authors would like to thank Doctor Nguyen Xuan Thanh for taking TEM images.

\section{References}

[1] M. Ekor, "The growing use of herbal medicines: issues relating to adverse reactions and challenges in monitoring safety," Frontiers in Pharmacology, vol. 4, p. 177, 2014.

[2] S. Ansari, F. Islam, and M. Sameem, "Influence of nanotechnology on herbal drugs: a review," Journal of advanced pharmaceutical technology, vol. 3, no. 3, p. 142, 2012.

[3] T. Gunasekaran, T. Haile, T. Nigusse, and M. D. Dhanaraju, "Nanotechnology: an effective tool for enhancing bioavailability and bioactivity of phytomedicine," Asian Pacific Journal of Tropical Biomedicine, vol. 4, Suppl 1, pp. S1-S7, 2014.

[4] A. P. Nikam, M. P. Ratnaparkhiand, and S. P. Chaudhari, "Nanoparticles-an overview," International Journal of Research Development in Pharmacy Life Sciences, vol. 3, no. 5, pp. 1121-1127, 2014.

[5] P. N. Le and N. Q. Tran, "Advances in thermosensitive polymer-grafted platforms for biomedical applications," Materials Science and Engineering C, vol. 92, pp. 1016-1030, 2018.

[6] P. N. Le, D. C. Pham, D. H. Nguyen et al., "Poly (N-isopropylacrylamide)-functionalized dendrimer as a thermosensitive nanoplatform for delivering Malloapelta B against HepG2 cancer cell proliferation," Advances in Natural Sciences: Nanoscience Nanotechnology, vol. 8, no. 2, p. 025014, 2017.

[7] D. Van Thoai, D. T. Nguyen, N. H. Nguyen et al., "Lipophilic effect of various pluronic-grafted gelatin copolymers on the quercetin delivery efficiency in these self-assembly nanogels," Journal of Polymer Research, vol. 27, no. 12, pp. 1-12, 2020.

[8] V. P. Torchilin, "Block copolymer micelles as a solution for drug delivery problems," Expert Opinion on Therapeutic Patents, vol. 15, no. 1, pp. 63-75, 2005.

[9] D. T. Nguyen, V. T. Dinh, L. H. Dang et al., "Dual interactions of amphiphilic gelatin copolymer and nanocurcumin improv- ing the delivery efficiency of the nanogels," Polymers, vol. 11, no. 5 , p. 814, 2019.

[10] A. M. Butt, M. C. I. M. Amin, H. Katas, N. Sarisuta, W. Witoonsaridsilp, and R. Benjakul, "In Vitro Characterization of Pluronic F127 and D- -Tocopheryl Polyethylene Glycol 1000 Succinate Mixed Micelles as Nanocarriers for Targeted Anticancer- Drug Delivery," Journal of Nanomaterials, vol. 2012, Article ID 916573, 11 pages, 2012.

[11] P. J. Sheskey, W. G. Cook, and C. G. Cable, Handbook of Pharmaceutical Excipients, Pharmaceutical Press, UK, 8 edition edition, 2017.

[12] G. Dumortier, J. L. Grossiord, F. Agnely, and J. C. Chaumeil, "A review of poloxamer 407 pharmaceutical and pharmacological characteristics," Pharmaceutical Research, vol. 23, no. 12, pp. 2709-2728, 2006.

[13] M. Ali-Seyed and K. Vijayaraghavan, "Nutraceuticals for Wound Healing: A Special Focus on Chromolaena odorata as Guardian of Health with Broad Spectrum of Biological Activities," in Nutraceuticals in Veterinary Medicine, R. C. Gupta, A. Srivastava, and R. Lall, Eds., pp. 541-562, Springer, Cham, Switzerland, 2019.

[14] A. Suksamrarn, A. Chotipong, T. Suavansri et al., "Antimycobacterial activity and cytotoxicity of flavonoids from the flowers of Chromolaena odorata," Archives of Pharmacal Research, vol. 27, no. 5, pp. 507-511, 2004.

[15] E. H. Heiss, T. V. A. Tran, K. Zimmermann et al., "Identification of chromomoric acid CI as an Nrf2 activator in Chromolaena odorata," Journal of Natural Products, vol. 77, no. 3, pp. 503-508, 2014.

[16] O. Irobi, "Activities of_Chromolaena odorata_(Compositae) leaf extract against_Pseudomonas aeruginosa_and_Streptococcus faecalis_," Journal of Ethnopharmacology, vol. 37, no. 1, pp. 81-83, 1992.

[17] M. C. Stanley, O. E. Ifeanyi, C. C. Nwakaego, and I. O. Esther, "Antimicrobial effects of Chromolaena odorata on some human pathogens," International Journal of Current Microbiology and Applied Sciences, vol. 3, no. 3, pp. 1006-1012, 2014.

[18] P. B. K. Kouamé, C. Jacques, G. Bedi et al., "Phytochemicals isolated from leaves of Chromolaena odorata: impact on viability and clonogenicity of cancer cell lines," Phytotherapy Research, vol. 27, no. 6, pp. 835-840, 2013.

[19] A. A. Adedapo, A. A. Oyagbemi, O. A. Fagbohun, T. O. Omobowale, and M. A. Yakubu, "Evaluation of the anticancer properties of the methanol leaf extract of Chromolaena odorata on HT-29 cell line," Journal of Pharmacognosy and Phytochemistry, vol. 5, no. 2, p. 52, 2016.

[20] L. Amazu, P. Omoregie, A. Ajugwo, C. Ifezulike, and C. Azikiwe, "Anticonvulsant potency of the leaf extract of Chromolaena odorata in rats," Unique Research Journal of Medicine and Medical Sciences, vol. 1, pp. 64-69, 2013.

[21] M. Onkaramurthy, V. Veerapur, B. Thippeswamy, T. M. Reddy, H. Rayappa, and S. Badami, "Anti-diabetic and anticataract effects of _Chromolaena odorata_ Linn., in streptozotocin-induced diabetic rats," Journal of Ethnopharmacology, vol. 145, no. 1, pp. 363-372, 2013.

[22] F. O. Uhegbu, C. Imo, and C. H. Onwuegbuchulam, "Lipid lowering, hypoglycemic and antioxidant activities of Chromolaena odorata (L) and Ageratum conyzoides (L) ethanolic leaf extracts in albino rats," Journal of Medicinal Plants Studies, vol. 4, no. 2, pp. 155-159, 2016. 
[23] S. Ijioma, A. Okafor, P. Ndukuba, A. Nwankwo, and S. Akomas, "Hypoglycemic, hematologic and lipid profile effects of Chromolaena odorata ethanol leaf extract in alloxan induced diabetic rats," Annals of Biological Sciences, vol. 2, pp. 27-32, 2014.

[24] M. Atindehou, L. Lagnika, B. Guérold et al., "Isolation and identification of two antibacterial agents from \&lt;i\&gt;Chromolaena odorata\&lt;/i\&gt; L. active against four diarrheal strains," Advances in Microbiology, vol. 3, no. 1, pp. 115-121, 2013.

[25] P. E. Aba, P. E. Joshua, F. C. Ezeonuogu, M. I. Ezeja, V. U. Omoja, and P. U. Umeakuana, "Possible anti-diarrhoeal potential of ethanol leaf extract of Chromolaena odorata in castor oil-induced rats," Journal of Complementary and Integrative Medicine, vol. 12, no. 4, pp. 301-306, 2015.

[26] A. N. Ngane, R. E. Etame, F. Ndifor, L. Biyiti, P. A. Zollo, and P. Bouchet, "Antifungal activity of $<\mathrm{i}>$ Chromolaena odorata $</ \mathrm{i}>$ (L.) King \& Robinson(Asteraceae) of Cameroon," Chemotherapy, vol. 52, no. 2, pp. 103-106, 2006.

[27] K. Naidoo, R. Coopoosamy, and G. Naidoo, "Screening of Chromolaeana odorata (L.) King and Robinson for antibacterial and antifungal properties," Journal of Medicinal Plants Research, vol. 5, no. 19, pp. 4859-4862, 2011.

[28] V. B. Owoyele, J. O. Adediji, and A. O. Soladoye, "Anti-inflammatory activity of aqueous leaf extract of Chromolaena odorata," Inflammopharmacology, vol. 13, no. 5-6, pp. 479-484, 2005.

[29] T. T. H. Hanh, D. T. T. Hang, C. Van Minh, and N. T. Dat, "Anti-inflammatory effects of fatty acids isolated from Chromolaena odorata," Asian Pacific Journal of Tropical Medicine, vol. 4, no. 10, pp. 760-763, 2011.

[30] H. Pandith, X. Zhang, S. Thongpraditchote, Y. Wongkrajang, W. Gritsanapan, and S. J. Baek, "Effect of Siam weed extract and its bioactive component scutellarein tetramethyl ether on anti-inflammatory activity through NF- $\kappa$ B pathway," Journal of Ethnopharmacology, vol. 147, no. 2, pp. 434-441, 2013.

[31] P. T. Thang, L. S. Teik, and C. S. Yung, "Anti-oxidant effects of the extracts from the leaves of _Chromolaena odorata_ on human dermal fibroblasts and epidermal keratinocytes against hydrogen peroxide and hypoxanthine -xanthine oxidase induced damage," Burns, vol. 27, no. 4, pp. 319-327, 2001.

[32] A. C. Akinmoladun, E. Ibukun, and I. Dan-Ologe, "Phytochemical constituents and antioxidant properties of extracts from the leaves of Chromolaena odorata," Scientific Research and Essays, vol. 2, no. 6, pp. 191-194, 2007.

[33] K. P. Melinda, X. Rathinam, K. Marimuthu et al., "A comparative study on the antioxidant activity of methanolic leaf extracts of Ficus religiosa L, Chromolaena odorata (L.) King \& Rabinson, Cynodon dactylon (L.) Pers. and Tridax procumbens L.," Asian Pacific Journal of Tropical Medicine, vol. 3, no. 5, pp. 348-350, 2010.

[34] K. S. Rao, P. K. Chaudhury, and A. Pradhan, "Evaluation of anti-oxidant activities and total phenolic content of_Chromolaena odorata_," Food and Chemical Toxicology, vol. 48, no. 2, pp. 729-732, 2010.

[35] K. Vijayaraghavan, A. Mohammed, and R. Maruthi, "Studies on phytochemical screening \& antioxidant activity of Chromolaena odorata \& Anonasquamosal," International Journal of Innovative Research in Science, Engineering \&Technology, vol. 2, no. 12, 2013.

[36] T. Boudjeko, R. Megnekou, A. L. Woguia et al., "Antioxidant and immunomodulatory properties of polysaccharides from
Allanblackia floribunda Oliv stem bark and Chromolaena odorata (L.) King and H.E. Robins leaves," BMC research notes, vol. 8, no. 1, p. 759, 2015.

[37] I. Ezenyi, O. Salawu, R. Kulkarni, and M. Emeje, "Antiplasmodial activity-aided isolation and identification of quercetin-4'methyl ether in Chromolaena odorata leaf fraction with high activity against chloroquine-resistant Plasmodium falciparum," Parasitology Research, vol. 113, no. 12, pp. 44154422, 2014.

[38] M. Boppré, T. Thoden, and J. Hallmann, "Pyrrolizidine alkaloids of Chromolaena odorata act as nematicidal agents and reduce infection of lettuce roots by Meloidogyne incognita," Nematology, vol. 9, no. 3, pp. 343-349, 2007.

[39] A. Pandurangan, K. Rana, and A. Singh, "Evaluation wound healing activity of leaves of Chromolaena odorata Linn," International Journal of Pharmaceutical Sciences Letters, vol. 5, pp. 555-557, 2015.

[40] G. Anyasor, D. Aina, M. Olushola, and A. Aniyikaye, "Phytochemical constituent, proximate analysis, antioxidant, antibacterial and wound healing properties of leaf extracts of Chromolaena odorata," Annals of Biological Research, vol. 2, no. 2, pp. 441-451, 2011.

[41] P. Akah, "Mechanism of hemostatic activity ofEupatorium odoratum," International Journal of Crude Drug Research, vol. 28, no. 4, pp. 253-256, 2008.

[42] H. Pandith, S. Thongpraditchote, Y. Wongkrajang, and W. Gritsanapan, "In vivo and in vitro hemostatic activity of Chromolaena odorata leaf extract," Pharmaceutical Biology, vol. 50, no. 9, pp. 1073-1077, 2012.

[43] C. S. Alisi, G. O. C. Onyeze, O. A. Ojiako, and C. G. Osuagwu, "Evaluation of the protective potential of Chromolaena odorata Linn. extract on carbon tetrachloride-induced oxidative liver damage," International Journal of Biochemistry Research \& Review, vol. 1, no. 3, p. 69, 2011.

[44] R. Asomugha, P. Okafor, I. Ijeh, O. Orisakwe, and A. Asomugha, "Hepatic effects of aqueous extract of Chromolaena odorata in male Wistar albino rats," Pharmacology, vol. 1, pp. 127-136, 2014.

[45] Council for International Organization of Medical Sciences and the International Council for Laboratory Animal Science, "The International Guiding Principles for Biomedical Research Involving Animals," 2012, https://olaw.nih.gov/ sites/default/files/Guiding_Principles_2012.pdf.

[46] Y.-C. Kuo and J.-F. Chung, "Physicochemical properties of nevirapine-loaded solid lipid nanoparticles and nanostructured lipid carriers," Colloids and Surfaces B: Biointerfaces, vol. 83, no. 2, pp. 299-306, 2011.

[47] V. L. Singleton, R. Orthofer, and R. M. Lamuela-Raventós, "Analysis of total phenols and other oxidation substrates and antioxidants by means of folin-ciocalteu reagent," Methods in Enzymology, vol. 299, pp. 152-178, 1999.

[48] P. Skehan, R. Storeng, D. Scudiero et al., "New colorimetric cytotoxicity assay for anticancer-drug screening," JNCI Journal of the National Cancer Institute, vol. 82, no. 13, pp. 1107-1112, 1990.

[49] A. Rajasekaran, M. Kalaivani, and G. Ariharasivakumar, "Haemostatic effect of fresh juice and methanolic extract of. leaves in rat model," International Journal of Biological and Medical Research, vol. 1, no. 3, pp. 85-87, 2010.

[50] C. Ghai, A Textbook of Practical Physiology, JP Medical Ltd, New Delhi, India, 8 edition edition, 2012. 
[51] R. Basak and R. Bandyopadhyay, "Encapsulation of hydrophobic drugs in Pluronic F127 micelles: effects of drug hydrophobicity, solution temperature, and $\mathrm{pH}$," Langmuir, vol. 29, no. 13, pp. 4350-4356, 2013.

[52] P. K. Sharma and S. R. Bhatia, "Effect of anti-inflammatories on Pluronic ${ }^{\oplus}$ F127: micellar assembly, gelation and partitioning," International Journal of Pharmaceutics, vol. 278, no. 2, pp. 361-377, 2004.

[53] L. H. Dang, M. T. Vu, J. Chen et al., "Effect of ultrasonication on self-assembled nanostructures formed by amphiphilic positive-charged copolymers and negative-charged drug," ACSomega, vol. 4, no. 3, pp. 4540-4552, 2019.

[54] L. Shang, K. Nienhaus, and G. U. Nienhaus, "Engineered nanoparticles interacting with cells: size matters," Journal of nanobiotechnology, vol. 12, no. 1, p. 5, 2014.

[55] K. Y. Win and S.-S. Feng, "Effects of particle size and surface coating on cellular uptake of polymeric nanoparticles for oral delivery of anticancer drugs," Biomaterials, vol. 26, no. 15, pp. 2713-2722, 2005.

[56] H. S. Kang, S.-S. Kwon, Y.-S. Nam, S.-H. Han, and I.-S. Chang, "Determination of zeta potentials of polymeric nanoparticles by the conductivity variation method," Journal of Colloid and Interface Science, vol. 255, no. 2, pp. 352-355, 2002.

[57] J. Zhang, D. Wang, Y. Wu et al., "Lipid-polymer hybrid nanoparticles for oral delivery of tartary buckwheat flavonoids," Journal of Agricultural and Food Chemistry, vol. 66, no. 19, pp. 4923-4932, 2018.

[58] M. Soleimanifar, S. M. Jafari, and E. Assadpour, "Encapsulation of olive leaf phenolics within electrosprayed whey protein nanoparticles; production and characterization," Food Hydrocolloids, vol. 101, p. 105572, 2020.

[59] M.-H. Nguyen, S. E. Lee, T.-T. Tran et al., "A simple strategy to enhance the in vivo wound-healing activity of curcumin in the form of self-assembled nanoparticle complex of curcumin and oligochitosan," Materials Science and Engineering: C, vol. 98, pp. 54-64, 2019.

[60] T. T. Phan, M. A. Hughes, and G. W. Cherry, "Enhanced proliferation of fibroblasts and endothelial cells treated with an extract of the leaves of Chromolaena odorata (Eupolin), an herbal remedy for treating wounds," Plastic and Reconstructive Surgery, vol. 101, no. 3, pp. 756-765, 1998.

[61] J. Qu, X. Zhao, Y. Liang, T. Zhang, P. X. Ma, and B. Guo, "Antibacterial adhesive injectable hydrogels with rapid selfhealing, extensibility and compressibility as wound dressing for joints skin wound healing," Biomaterials, vol. 183, pp. 185-199, 2018.

[62] J. He, M. Shi, Y. Liang, and B. Guo, "Conductive adhesive selfhealing nanocomposite hydrogel wound dressing for photothermal therapy of infected full-thickness skin wounds," Chemical Engineering Journal, vol. 394, p. 124888, 2020.

[63] H. Pandith, X. Zhang, J. Liggett, K.-W. Min, W. Gritsanapan, and S. J. Baek, "Hemostatic and wound healing properties of Chromolaena odorata leaf extract," ISRN Dermatology, vol. 2013, 8 pages, 2013.

[64] S. Akomas and S. Ijioma, "Bleeding and clotting time effect of ethanolic extracts of Chromolaena odorata versus Ocimum gratissimum treated albino rats," Journals of Medical Sciences, vol. 2, no. 1, pp. 9-13, 2014 